\title{
Modelo basado en Agentes para la Detección de Fallas Cognitivas en Entornos de Aprendizaje Colaborativo
}

\author{
Santiago Álvarez, Oscar M. Salazar, Demetrio A. Ovalle \\ Universidad Nacional de Colombia, Departamento de Ciencias de la Computación y de la Decisión, \\ Facultad de Minas, Medellín-Colombia (e-mail: salvarezl@unal.edu.co,omsalazaro@unal.edu.co, \\ dovalle@unal.edu.co)
}

Recibido Ene. 23, 2018; Aceptado Mar. 26, 2018; Versión final May. 23, 2018, Publicado Oct. 2018

\begin{abstract}
Resumen
El objetivo de este artículo es proponer y validar un modelo basado en la integración de diferentes técnicas de la Inteligencia Artificial, como ontologías y agentes inteligentes de software con el fin de solventar problemáticas que aún persisten en el aprendizaje colaborativo. La metodología empleada incluye una representación de conocimientos basada en un modelo ontológico, el cual permite generar inferencias para la recomendación más acertada de recursos educativos a partir de las fallas cognitivas detectadas en cada estudiante durante el desarrollo de una actividad colaborativa. Adicionalmente, el modelo incorpora agentes inteligentes con el fin de brindar características de adaptabilidad, distribución de tareas y proactividad en los procesos de recomendación de recursos educativos. La validación del modelo propuesto fue realizada a través de la implementación de un prototipo aplicado a casos de estudio. Los resultados obtenidos evidencian los beneficios de integrar ontologías y agentes inteligentes para detectar fallas cognitivas en entornos de aprendizaje colaborativo.
\end{abstract}

Palabras clave: detección de fallas cognitivas; aprendizaje colaborativo; agentes inteligentes; ontologías

\section{Agent-based Model for Cognitive Failure Detection in Collaborative Learning Environments}

\begin{abstract}
The aim of this paper is to propose and validate a model based on the integration of several artificial intelligence techniques, such as ontologies and intelligent software agents in order to face problems that still persist in collaborative learning. The methodology employed includes a knowledge representation based on an ontological model, which allows generating inferences in order to produce most accurate recommendation of educational resources based on cognitive failures detected on each student during a collaborative activity. In addition, the model incorporates intelligent agents to provide characteristics of adaptivity, task distribution, and proactivity in educational resources recommendation processes. The validation of the proposed model was done through the implementation of a prototype applied to case studies. The results show the benefits of integrating ontologies and intelligent agents to detect cognitive failure in collaborative learning processes.
\end{abstract}

Keywords: cognitive failure detection; collaborative learning; intelligent agents; ontologies 
Modelo basado en Agentes para la Detección de Fallas Cognitivas en Entornos de Aprendizaje _ Álvarez

\section{INTRODUCCIÓN}

En los últimos años el aprendizaje colaborativo apoyado por computador o CSCL (por sus siglas en inglés para Computer Supported Collaborative Learning) ha sido impulsado por los profesores al interior de las aulas de clase con el objetivo de ampliar y diversificar los procesos de adquisición y generación de nuevos conocimientos, aprovechando así las ventajas brindadas por los entornos de aprendizaje colaborativo. Adicionalmente, se busca que los estudiantes organizados en equipos trabajen juntos para lograr un objetivo común y además puedan discutir desde diferentes puntos de vista, siempre buscando mejorar los procesos de aprendizaje. Por otro lado, Herrera et al. (2017) afirman "el trabajo en equipo es una habilidad fundamental que todos los ingenieros deben tener en su carrera profesional" (p.50). Los procesos de CSCL incluyen diferentes etapas como la formación de grupos, la planificación de tareas de trabajo en equipo, la asignación de recursos de aprendizaje, la evaluación del trabajo en equipo, la evaluación de tareas, entre otros (Hübscher, 2010). La noción de ontología surge de la necesidad de representar formal y semánticamente el conocimiento utilizado en un dominio de aplicación con el fin de realizar inferencias entre los conceptos involucrados (de Graaf et al., 2014), así como de permitir su disponibilidad, accesibilidad y reutilización en Internet a través de sistemas de recuperación y buscadores de información en la Web.

Por su parte, los agentes inteligentes corresponden a entidades computacionales autónomas y con capacidad para ejecutar tareas y lograr sus metas con un mínimo de supervisión humana o incluso ninguna. Para Caicedo y Weiss (2011) son características que deberían poseer los agentes para trabajar efectivamente: a) reactividad: responden inmediatamente a los cambios percibidos en su entorno; b) distribución de tareas: cada agente tiene funcionalidades bien definidas e identifica los problemas a resolver; c) proactividad: los agentes toman iniciativa para la solución de problemas; d) cooperación y coordinación: realizan tareas por medio de intercambio de mensajes con otros agentes a través de un lenguaje común; e) autonomía: los agentes no requieren de la intervención directa de los seres humanos para operar; f) deliberación: cada agente tiene la capacidad de realizar procesos de razonamiento interno los cuales le permitan tomar decisiones; g) movilidad: pueden moverse de un nodo a otro a través de la red; $h$ ) adaptación: a partir de los cambios en el entorno cambian su comportamiento y mejoran de esta forma su desempeño, y finalmente i) paralelismo: el sistema puede mejorar su desempeño a través de la ejecución de tareas realizadas en paralelo por los agentes. De esta forma, los sistemas multi-agente (SMA) se conforman a partir de un conjunto de agentes inteligentes que trabajan conjuntamente e interactúan coordinadamente en un entorno computacional para resolver problemas específicos y de alta complejidad. Según Ovalle et al. (2014) este tipo de sistemas ofrecen una gran facilidad de adquisición y procesamiento de información que puede encontrarse altamente distribuida. Así, este paradigma ofrece nuevas maneras para analizar, diseñar e implementar sistemas de software complejos (Carrera et al., 2014). Adicionalmente, se ha utilizado en el desarrollo de sistemas de recomendación (Rodríguez et al., 2016) que buscan proporcionar resultados de búsquedas teniendo en cuenta las necesidades y características cognitivas particulares de los usuarios. En el caso del e-learning (Acevedo et al., 2010) estos sistemas buscan ofrecer recomendaciones de recursos educativos de acuerdo a las características, gustos y necesidades del estudiante.

El trabajo "edutab box" propuesto por Oshima et al. (2016) propone una herramienta de aprendizaje colaborativo soportado por computador para cursos escolares de niveles inferiores. El estudio se centra en validar la usabilidad de la herramienta y en aclarar la contribución que esta tiene en el proceso de enseñanza a partir del despliegue de las funcionalidades. El objetivo de la aplicación es que los estudiantes por medio de dispositivos móviles -en este caso tablets-, puedan interactuar entre ellos y llevar a cabo una serie de actividades que se despliegan en el tablero. Actividades tales como: realizar pantallazos, seleccionar asignaturas favoritas y sustentarlo, tomar fotos de elementos pertenecientes a la clase, realizar cuestionarios, etc. La herramienta se apoya en un modelo colaborativo, que permite la difusión de resultados por parte de los estudiantes, lo que fomenta la discusión argumentativa. Sin embargo, la investigación no considera aspectos de detección de fallas, ni presenta una estructura de conocimiento explícita que permita describir mejor el modelo utilizado. Adicionalmente, es importante incluir nuevos recursos que respondan a las necesidades de los usuarios, dotando al sistema con características de adaptabilidad.

Por otra parte, Terzidou et al. (2016) proponen la implementación de características de Inteligencia Artificial sobre un juego serio para un entorno colaborativo aplicado a la educación terciaria. La investigación se encuentra basada en un trabajo previo realizado con agentes pedagógicos (Terzidou y Tsiatsos, 2016) los cuales son considerados como una técnica de aprendizaje colaborativo. El objetivo del estudio es determinar si el uso de herramientas de Inteligencia Artificial aplicado al desarrollo de juegos serios colaborativos es eficaz sobretodo en términos de lograr un mejor desempeño del equipo. Lo anterior se puede lograr si se mejoran las interacciones colaborativas de los estudiantes a través de mecanismos de motivación ofrecidos por el ambiente de aprendizaje. El juego está basado en un entorno virtual en 3D donde los estudiantes pueden interactuar y trabajar en equipo para ser campeones de una partida, la cual cuenta con un tiempo, y consiste en una exploración de un entorno donde los estudiantes tratan de descubrir objetos virtuales que 
contienen preguntas de selección múltiple. Las características de Inteligencia Artificial propuestas para apoyar el juego serio son las siguientes: un agente de juego pedagógico, personajes que no juegan y funcionan como guías para el juego virtual, robots de chat y una interfaz de juego llamada "Mapa de Progreso". El juego colaborativo cuenta con realimentación individual al estudiante, con seguimiento a la actividad y motiva a los estudiantes en el uso de la herramienta. Sin embargo, la propuesta no cuenta con un esquema de actividades que motive la interacción constante y a conciencia con los demás integrantes del equipo. Finalmente, tampoco se contempla una estructura para la detección de fallas cognitivas y la recomendación de recursos educativos adaptados a las necesidades de cada usuario.

En otra aproximación, Lescano et al. (2016) proponen un algoritmo genético para la formación automática de grupos considerando los estilos individuales de aprendizaje de los estudiantes. Para ello, se utilizaron los datos históricos sobre el rendimiento de los grupos para crear reglas de asociación, que se emplean posteriormente en la función de fitness. El algoritmo diseña diferentes alternativas de agrupación considerando los estilos de aprendizaje de los estudiantes bajo la restricción de producir grupos de tamaño equilibrado. El algoritmo propuesto fue analizado con diferentes tamaños de grupos suministrados por el profesor. A través de la experimentación, se puede inferir qué tipo de configuración se logra para ser más apropiado. Aunque este enfoque produce buenos resultados, no se consideran las falencias y el desempeño de los estudiantes, lo que puede ser un factor determinante en la conformación de equipos de trabajo.

Los trabajos anteriores presentan fortalezas significativas que permiten brindar información sobre los desarrollos que han tenido lugar dentro del campo de CSCL. Sin embargo, estas investigaciones también muestran algunas debilidades que deben afrontarse para mejorar los procesos de aprendizaje colaborativo. Así, por ejemplo, no consideran la detección temprana de fallas cognitivas, la realización de evaluaciones constantes durante el proceso de aprendizaje, la detección de fortalezas detectadas en los estudiantes y la recomendación de recursos educativos en el desarrollo de las actividades colaborativas con el objetivo de corregir las fallas cognitivas identificadas. Adicionalmente, persisten problemáticas en los procesos de aprendizaje colaborativo tales como: 1) generalmente se evalúa a todos los estudiantes del equipo por igual; 2) no se realizan evaluaciones constantes durante las actividades para detectar fallas cognitivas tempranas; 3 ) los modelos actuales de aprendizaje colaborativo no recomiendan recursos educativos para solventar las fallas cognitivas identificadas.

Con el fin de solventar problemáticas que aún persisten en área del CSCL el objetivo de este artículo es proponer y validar un modelo de aprendizaje colaborativo basado en la integración de diferentes técnicas de la Inteligencia Artificial (IA), como las ontologías y los agentes inteligentes de software. La incorporación de una ontología de dominio específico permite la representación semántica de las entidades relacionadas con el esquema de desarrollo de actividades colaborativas y permite generar inferencias para la recomendación de recursos educativos. Por otro lado, el uso de agentes inteligentes permite la incorporación de funcionalidades de sensibilidad al contexto, es decir, se utilizan características del estudiante y del entorno en el que este se desenvuelve para mejorar la identificación de fallas cognitivas y así ofrecer una recomendación de recursos educativos proactiva que permita corregir dichas fallas.

\section{DESARROLLO DEL MODELO}

Como ya se ha mencionado, el modelo de aprendizaje colaborativo considera la integración de diferentes técnicas de la IA, como las ontologías y los agentes inteligentes de software. Es por ello, que el desarrollo del modelo considera tres etapas: A) la identificación de las actividades colaborativas y el desarrollo de un esquema para el desarrollo de las mismas; B) el proceso de especificación y el desarrollo del modelo ontológico; y C) la arquitectura multi-agente para el despliegue del sistema colaborativo. A continuación, se detallan cada una de las etapas.

\section{Esquema para el desarrollo de actividades colaborativas}

Para el proceso de detección de fallas cognitivas se propone un esquema secuencial, el cual se encuentra basado en el método de aprendizaje JigSaw (Esnawy, 2016). Este, busca potenciar el aprendizaje colaborativo a través de la integración de una serie de actividades bien definidas, permitiendo así la realimentación al estudiante y la recomendación de recursos educativos enfocados en la corrección de las fallas cognitivas identificadas durante las etapas del flujo. Teniendo en cuenta las limitaciones encontradas en trabajos previos se propone un modelo de aprendizaje colaborativo que incluye las siguientes funcionalidades: 1) especificación formal de fallas cognitivas; 2) detección de fallas cognitivas en las etapas de evaluación de conocimientos; 3) recomendación de recursos educativos a partir de las fallas detectadas; y 4) reconformación de equipos para superar las debilidades (correspondientes a las fallas cognitivas identificadas). La figura 1 presenta las etapas inteligentes agrupadas según las siguientes convenciones: (A) Actividad colaborativa y evaluación de la misma; (B) Diagnóstico de fallas cognitivas; (C) Realimentación, 
recomendación de recursos educativos y presentación de estadísticas al profesor; (D) Reconformación de equipos, caracterización de fortalezas y fallas de cada estudiante; y (E) Actividad lúdica de apoyo al aprendizaje colaborativo. Las etapas del esquema mostrado en la Figura 1 se describen en la Tabla 1.

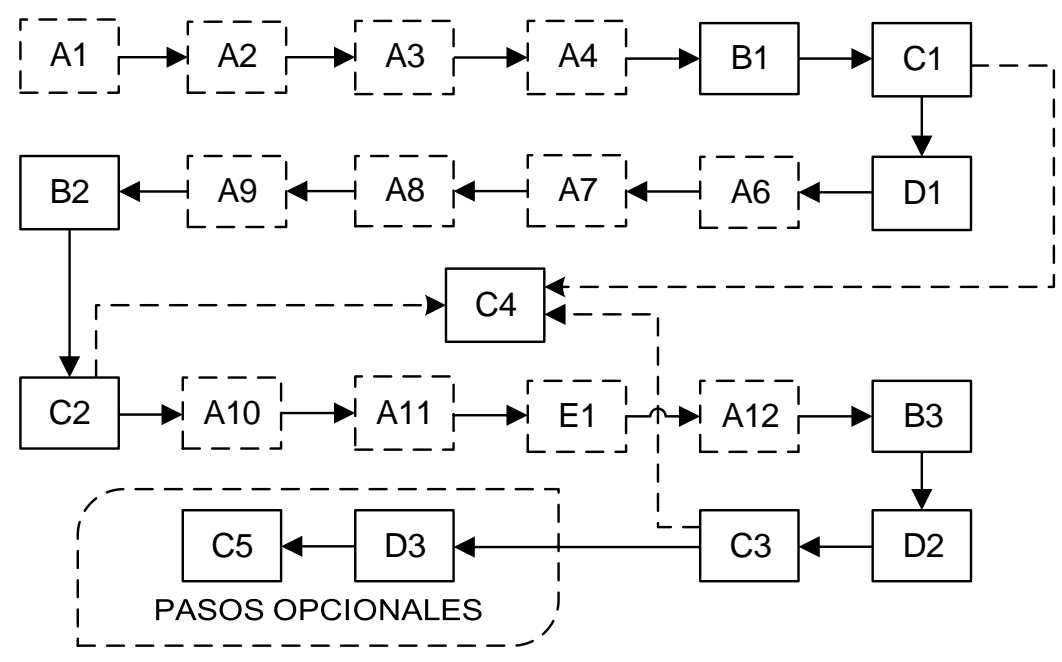

Fig. 1: Diagrama de Flujo para el desarrollo de actividades colaborativas extendido del método de aprendizaje JigSaw (Esnawy, 2016).

Tabla 1: Descripción de las actividades involucradas en las etapas del Flujo mostrado en la Figura 1.

\begin{tabular}{|c|c|}
\hline A1 & $\begin{array}{l}\text { ormación de equipos: ya que esta investigación no considera un modelo para la conformación de equipos, } \\
\text { ección de sus integrantes se deberá realizar sin tener en cuenta las características cognitivas de los } \\
\text { liantes y por equipo se deberá contar con un número de miembros entre } 4 \text { y } 6 \text { personas. }\end{array}$ \\
\hline $\bar{A} 2$ & $\begin{array}{l}\text { gnación de elementos de aprendizaje mutuamente excluyentes a cada integrante del equipo (igual para cada } \\
\text { ipo): en el ámbito de una temática, el profesor de forma previa construirá una actividad, la cual será divisible } \\
\text { pequeñas sub-actividades (llamados elementos de aprendizaje) relacionadas entre ellas, pero mutuamente } \\
\text { luyentes para su desarrollo. Dicha actividad será asignada a todos los equipos y cada elemento de } \\
\text { endizaje será asignado aleatoriamente a cada estudiante al interior de cada equipo. }\end{array}$ \\
\hline A3 & $\begin{array}{l}\text { sarrollo de la primera actividad individual de cada estudiante: en esta etapa cada estudiante ejecuta la } \\
\text { ividad correspondiente al elemento de aprendizaje que le fue asignado. }\end{array}$ \\
\hline A4 & $\begin{array}{l}\text { cada estudiante. Los resultados serán almacenados } \mathrm{p} \\
\text { sario en la siguiente etapa. }\end{array}$ \\
\hline $\begin{array}{l}\text { (B1; } \\
\text { B2; } \\
\text { B3) }\end{array}$ & (2) \\
\hline $\begin{array}{l}(\mathrm{C} 1 ; \\
\mathrm{C} 2 ; \\
\mathrm{C} 3)\end{array}$ & $\begin{array}{l}\text { allas } \\
\text { ción } \\
\text { os a } \\
\text { ción } \\
\text { vicio } \\
\text { e un } \\
\end{array}$ \\
\hline D1 & $\begin{array}{l}\text { una } \\
\text { on el } \\
\text { cada }\end{array}$ \\
\hline A6 & $\begin{array}{l}\text { Vo equipo } \\
\text { esquema } \\
\text { le fueran }\end{array}$ \\
\hline A7 & \\
\hline AO & \\
\hline A9 & ió haber adquirido luego de la reunión con los expertos. Además, los resultados \\
\hline
\end{tabular}


Tabla 1 (continuación)

\begin{tabular}{|c|c|}
\hline A10 & $\begin{array}{l}\text { Regreso de los estudiantes al equipo base: los estudiantes regresarán al equipo donde inicialmente comenzaron } \\
\text { con la actividad colaborativa (equipo base), y una vez allí se dará inicio a la siguiente etapa. }\end{array}$ \\
\hline A11 & $\begin{array}{l}\text { Divulgación de conocimientos al interior del equipo base por parte de cada estudiante: en esta etapa cada } \\
\text { estudiante -quién será experto en su elemento de aprendizaje- presentará de forma detallada mediante una } \\
\text { exposición los conocimientos adquiridos en las etapas anteriores. Dicha presentación será realizada a sus } \\
\text { compañeros de equipo base y tendrá la intención de transmitir todo el conocimiento posible. }\end{array}$ \\
\hline E1 & $\begin{array}{l}\text { Ejecución de actividad lúdica para evaluación de conocimientos: la actividad lúdica consiste en una serie de } \\
\text { pasos cuyo objetivo es apoyar e incentivar a los estudiantes en el proceso de aprendizaje colaborativo y se } \\
\text { constituye como se menciona a continuación: (i) los integrantes de cada equipo base construirán una serie de } \\
\text { preguntas con respuesta de alto nivel (aproximadamente } 3 \text { preguntas por estudiante); (ii) se selecciona un } \\
\text { estudiante aleatorio del equipo donde se construyó la pregunta, quién será conocido de aquí en adelante como } \\
\text { estudiante retador; (iii) se selecciona un estudiante aleatorio de un equipo adicional quién será conocido de aquí } \\
\text { en adelante como estudiante retado; (iv) según la dificultad de la pregunta, se contará con un tiempo para darle } \\
\text { la oportunidad al estudiante retado de responder correctamente y así ganar un punto para su equipo. En caso } \\
\text { de no responder o hacerlo de forma errada, el estudiante retador estará en la obligación de responder la } \\
\text { pregunta correctamente para evitar que su equipo pierda un punto; (v) la actividad finaliza cuando todos los } \\
\text { estudiantes hayan sido retados una vez, en dicho momento se asignará una nota a cada equipo de acuerdo a } \\
\text { la tabla de posiciones final generada a partir de los puntos. }\end{array}$ \\
\hline A12 & $\begin{array}{l}\text { Evaluación individual acumulativa (preguntas aleatorias): en esta etapa se realizará una evaluación individual la } \\
\text { cual contará con preguntas sobrantes de la actividad lúdica y con preguntas realizadas por el profesor. Una vez } \\
\text { más, los resultados serán almacenados para continuar con el procesamiento de la información necesaria para } \\
\text { la siguiente etapa. }\end{array}$ \\
\hline D2 & $\begin{array}{l}\text { Caracterización de fortalezas y fallas cognitivas de cada estudiante: el sistema realizará el procesamiento } \\
\text { necesario de la información capturada en el desarrollo de la actividad colaborativa para inferir fallas y fortalezas } \\
\text { cognitivas de cada estudiante. }\end{array}$ \\
\hline D3 & $\begin{array}{l}\text { Reconformación de equipos basada en fallas cognitivas identificadas: esta y la siguiente etapa son opcionales } \\
\text { y a criterio del profesor; si se desean realizar, el sistema propondrá una reconformación de equipos en la cual } \\
\text { estarán los estudiantes agrupados por fallas cognitivas similares e iguales. }\end{array}$ \\
\hline C5 & $\begin{array}{l}\text { Asignación de objetos de aprendizaje y tutores (integrantes del curso elegidos a partir de las fortalezas } \\
\text { identificadas en otros equipos): finalmente en esta etapa el sistema asignará recursos de aprendizaje (OAs y } \\
\text { tutores) a cada equipo según las fallas cognitivas identificadas. }\end{array}$ \\
\hline C4 & $\begin{array}{l}\text { Presentación de estadísticas al profesor: el sistema presentará durante esta etapa reportes estadísticos de } \\
\text { información capturada e inferida durante el desarrollo de la actividad colaborativa. Dichas estadísticas } \\
\text { corresponden a las siguientes: (i) visualización de las fallas cognitivas más repetidas; (ii) visualización de las } \\
\text { notas de la evaluación de cada estudiante; (iii) visualización de los estudiantes con mayor y menor número de } \\
\text { fallas cognitivas. }\end{array}$ \\
\hline
\end{tabular}

\section{Modelo de representación del conocimiento}

A partir del desarrollo de las fases de especificación, conceptualización e implementación pertenecientes a la metodología Methontology (Fernández-López et al., 1997; Valencia et al., 2017), se logró obtener el modelo ontológico presentado en la figura 2, el cual brinda inteligencia al modelo propuesto y conceptualiza formalmente los elementos asociados con el dominio. Este modelo presenta como entidad raíz la Lección del día desde la cual se despliega la Actividad propuesta por el profesor.

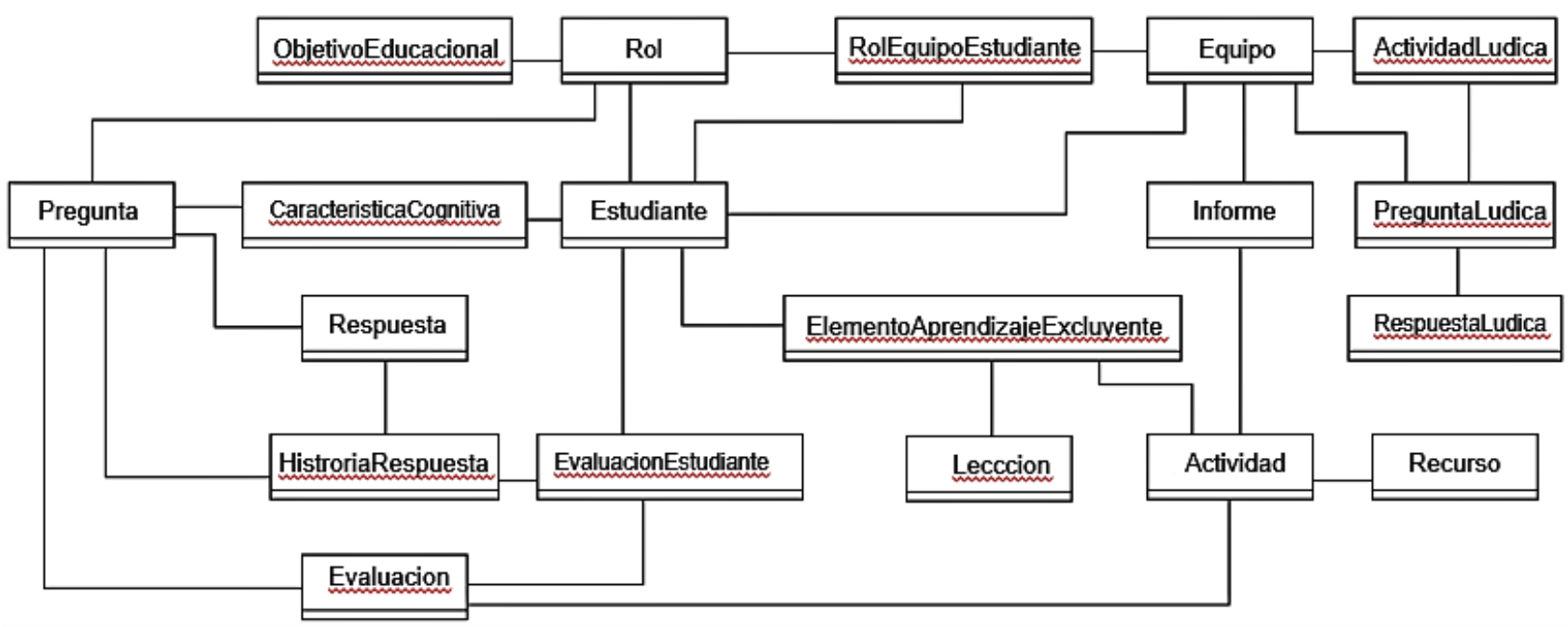

Fig. 2: Visualización de clases y relaciones de la ontología desarrollada. 
La Actividad se encuentra asociada con la entidad que contiene los Recursos de la misma, y con cada uno de los Elementos de Aprendizaje Mutuamente Excluyentes que son asignados a cada Estudiante en un Equipo. Por otro lado, existe una entidad que representa la Evaluación que a su vez está conformada por Preguntas y Respuestas. Finalmente, el modelo establece la estructura necesaria para almacenar tanto el conocimiento inherente a la descripción de las Características Cognitivas, así como toda la información asociada con la Actividad Lúdica propuesta en el esquema para el desarrollo de actividades colaborativas.

\section{Modelo multi-agente colaborativo}

La figura 3 presenta el modelo multi-agente propuesto en el cual se pueden observar las interacciones entre los agentes de software, las fuentes de información del sistema y los actores involucrados en el proceso de aprendizaje colaborativo y detección de fallas cognitivas del sistema. Este modelo es resultado de la aplicación de la metodología Prometheus (Lhafiane et al., 2015) utilizando sus tres fases definidas. Como actores externos se identificaron tres entidades: el profesor, el equipo de estudiantes y el sistema ROAp.

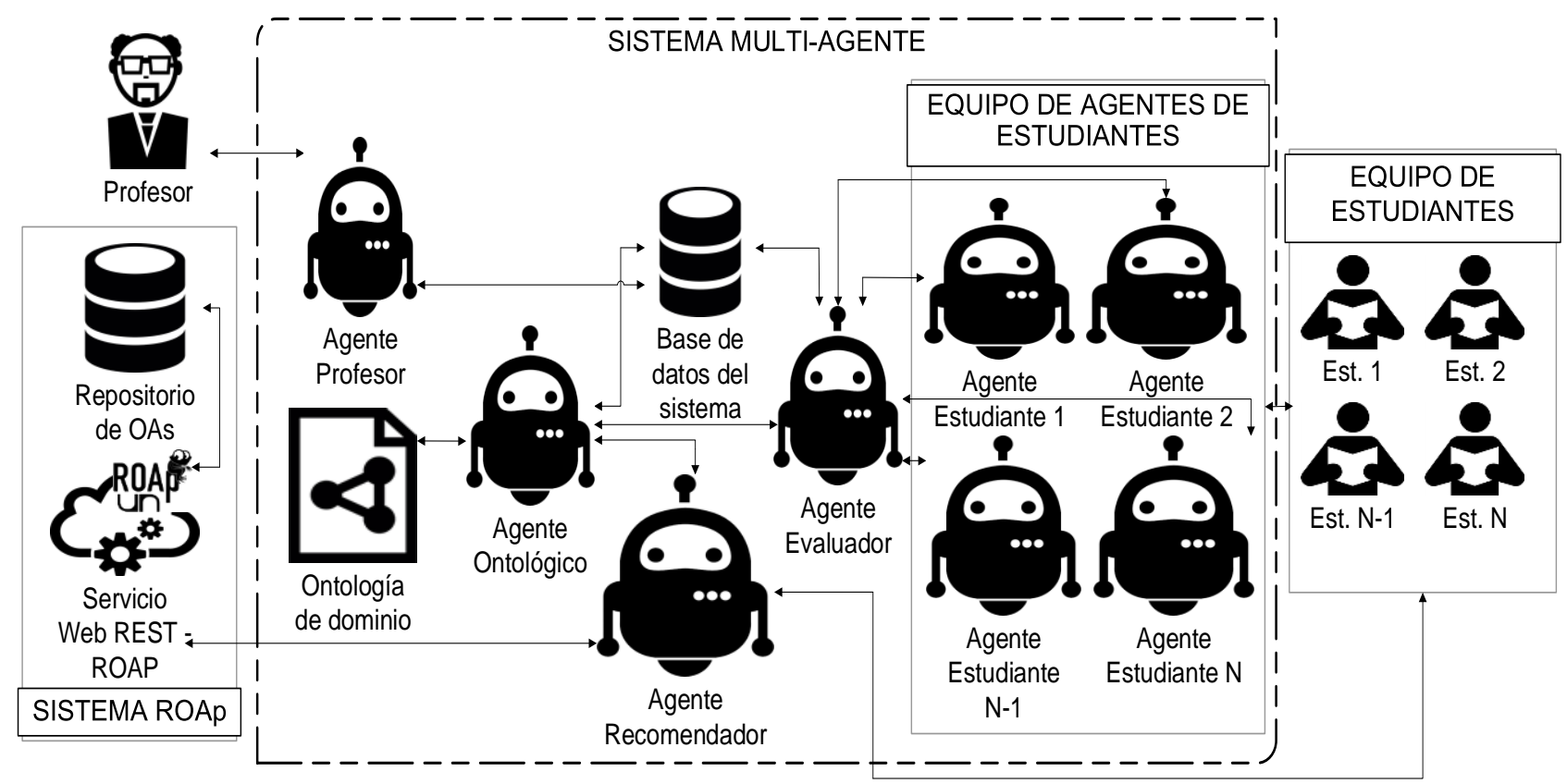

Fig. 3: Modelo propuesto de CSCL basado en agentes.

Adicionalmente, se definieron cinco tipologías de agentes, a saber: 1) Agente Estudiante: se encarga de representar al estudiante en el ámbito de la plataforma, así como de administrar su perfil. Es un agente de interfaz, que administra todas las interacciones (percepciones y/o acciones) entre el sistema y el estudiante; 2) Agente Profesor: representa al profesor dentro de la plataforma, lo asiste en el diseño instruccional de las actividades y presenta estadísticas de los resultados obtenidos por los estudiantes; 3) Agente Ontológico: administra la ontología de dominio específico, es decir, se encarga de poblar las entidades y de realizar inferencias solicitadas por otros agentes. Dichas inferencias son generadas a partir del uso de consultas y están relacionadas con la detección de fallas cognitivas, estructura de las actividades, recursos, información puntual de los estudiantes, detección de fallas cognitivas; 4) Agente Evaluador: despliega las evaluaciones a los estudiantes, transfiere la información y almacena los resultados en la BD (Base de Datos) del sistema; 5) Agente Recomendador: cumple dos funciones las cuales tienen como objetivo entregar información al Agente Estudiante para su posterior presentación, así: a) consumir el servicio Web expuesto por el sistema ROAp para obtener OAs. Dicho sistema recibe las palabras claves inferidas por el Agente Ontológico cuando se detectan fallas cognitivas; y b) obtener del Agente Ontológico los estudiantes que pueden cumplir el rol de tutores en la etapa final del esquema para el desarrollo de actividades colaborativas. Por su parte, las fuentes de información que maneja el sistema son las siguientes: 1) Ontología de dominio específico detallada previamente; 2) BD del sistema encargada de la persistencia de las entidades del sistema.

\section{DISCUSIÓN DE RESULTADOS}

La fase de implementación del modelo ontológico consiste en realizar una representación a través de un lenguaje formal, del conocimiento registrado en el proceso de desarrollo de las fases previas de especificación y conceptualización definidas por Methontology. Con el objetivo de desarrollar el modelo propuesto en esta investigación, se utilizó el lenguaje de Ontologías Web OWL mediante la herramienta Protégé (Tudorache et al., 2013). De esta manera, fueron creadas un total de 20 clases, 104 instancias, 30 propiedades de objetos 
y 51 propiedades de datos. Para la realimentación y la detección de fallas cognitivas asociadas a cada estudiante de los equipos, fueron creadas 2 reglas mediante el lenguaje de consulta SPARQL las cuales son presentadas en la Tabla 2 y explicadas a continuación de la Tabla 2.

Tabla 2: Reglas SPARQL para la realimentación y detección de fallas cognitivas.

\begin{tabular}{|c|c|}
\hline Regla1 & $\begin{array}{l}\text { SELECT ?estudiante ?evaluacion ?nota ?historialRespuesta ?respuesta ?esCorrecta ?palabrasClave } \\
\text { ?retroalimentacionPregunta WHERE \{ ?estudiante rdf:type ontology:Estudiante . ?estudiante } \\
\text { ontology:perteneceAEvaluacionEstudiante ?evaluacion . ?evaluacion ontology:notaEvaluacionEstudiante } \\
\text { ?nota. ?historialRespuesta rdf:type ontology:HistorialRespuesta . ?historialRespuesta } \\
\text { ontology:perteneceAEvaluacionEstudiante ?evaluacion . ?historialRespuesta ontology:tieneRespuesta } \\
\text { ?respuesta . ?respuesta ontology:esCorrectaRespuesta ?esCorrecta . ?respuesta } \\
\text { ontology:perteneceAPregunta ?pregunta . ?pregunta ontology:palabrasClavePregunta ?palabrasClave . } \\
\text { ?pregunta ontology:descripcionRetroalimentacionPregunta ?retroalimentacionPregunta . FILTER } \\
\text { (?nota < } 5 \text { \&\& ?esCorrecta = false) \} }\end{array}$ \\
\hline Regla2 & $\begin{array}{l}\text { SELECT ?estudiante ?evaluacion ?nota ?pregunta ?respuesta ?esCorrecta ?caracteristicaCognitiva } \\
\text { ?tipoCaracteristicaCognitiva WHERE \{ ?estudiante rdf:type ontology:Estudiante . ?estudiante } \\
\text { ontology:perteneceAEvaluacionEstudiante ?evaluacion . ?evaluacion ontology:notaEvaluacionEstudiante } \\
\text { ?nota.?historialRespuesta rdf:type ontology:HistorialRespuesta . ?historialRespuesta } \\
\text { ontology:perteneceAEvaluacionEstudiante ?evaluacion . ?historialRespuesta ontology:tieneRespuesta } \\
\text { ?respuesta . ?respuesta ontology:esCorrectaRespuesta ?esCorrecta . ?respuesta } \\
\text { ontology:perteneceAPregunta ?pregunta . ?pregunta ontology:detectaCaracteristicaCognitiva } \\
\text { ?caracteristicaCognitiva . } \\
\text { ?caracteristicaCognitiva ontology:tipoCaracteristicaCognitiva ?tipoCaracteristicaCognitiva . } \\
\text { FILTER (?nota < } 5 \text { \&\& ?esCorrecta = false \&\& sameTerm(?tipoCaracteristicaCognitiva, } \\
\text { "falla_cognitiva"^^xsd:string))\} }\end{array}$ \\
\hline
\end{tabular}

1) Regla1 (para la realimentación y recomendación de recursos): esta regla verifica las evaluaciones de cada estudiante y obtiene las palabras clave asociadas a las preguntas sobre las cuales su respuesta fue errada. Las palabras clave son utilizadas para la obtención de recursos educativos a través del consumo del servicio Web de ROAp mencionado anteriormente.

2) Regla2 (para la detección de fallas cognitivas): esta regla obtiene las fallas cognitivas de cada estudiante para utilizarlas en la presentación de estadísticas y la asociación con las palabras clave de cada pregunta.

Para el desarrollo de los agentes inteligentes propuestos se utiliza la metodología Prometheus (Lhafiane et al., 2015). Dicha metodología define las fases de especificación del sistema, diseño de la arquitectura y diseño detallado. A su vez, para la implementación del prototipo funcional se emplea el Framework JADE (Panisson et al., 2016). Este Framework, sigue los estándares definidos por FIPA (Foundation for Intelligent Agents) de la IEEE, se encuentra desarrollado para el lenguaje de programación JAVA y está orientado al desarrollo de SMA. Para el consumo del servicio Web necesario para la implementación de la funcionalidad de recomendación de recursos de aprendizaje, se hace uso del estándar RESTful obteniendo así las URL de cada uno de los recursos que son presentados al usuario en una interfaz Web HTML.

Respecto a la validación ontológica se simularon los perfiles de 5 estudiantes y falencias aleatorizadas correspondientes a dos de las temáticas abordadas en el módulo de Lógica Difusa tales como: deficiencia en la aplicación de operaciones sobre conjuntos difusos y falla en el método de inferencia difusa de Mamdani, las cuales fueron asignadas a cada estudiante. Posterior a ello, se ejecutaron las reglas anteriormente presentadas y se observó que el comportamiento de los resultados obtenidos era el esperado, ya que efectivamente se entregaba al usuario la información necesaria para cumplir con el objetivo de la ontología. Por otro lado, para realizar la validación del prototipo, se desplegó un caso de estudio en un ambiente controlado con 12 estudiantes que interactuaron con el sistema y recorrieron el flujo del esquema para el desarrollo de actividades colaborativas. En la figura 4, se presenta una de las interfaces del prototipo en donde el sistema identificó fallas cognitivas y muestra recomendaciones asociadas de recursos de educativos a cada uno de los estudiantes en las diferentes etapas del flujo de la actividad colaborativa. Adicionalmente, se realizaron encuestas de usabilidad, experiencia de usuario y efectividad de la recomendación de recursos basada en las fallas cognitivas identificadas.

Al comparar el enfoque presentado en este artículo con el de otros investigadores se puede afirmar lo siguiente: este trabajo de investigación incluye mecanismos de detección de fallas cognitivas; así mismo propone una estructura de representación de conocimientos bien definida basada en ontologías. Estos aspectos no son considerados por Oshima et al. (2016). Adicionalmente, este último trabajo no incluye la recomendación personalizada de recursos educativos, lo cual es una de las fortalezas de nuestro enfoque. 
Aunque el trabajo de Terzidou et al. (2016) utiliza agentes pedagógicos como el nuestro, no cuenta con un esquema de actividades que motive la interacción constante con los demás integrantes del equipo; tampoco se contempla una estructura para la detección de fallas cognitivas y no existe un mecanismo para la recomendación de recursos educativos adaptados a las necesidades de cada usuario, lo cual son puntos clave de nuestro enfoque. Finalmente, aunque nuestro enfoque no propone un mecanismo para formación automática de grupos considerando los estilos individuales de aprendizaje de los estudiantes como lo propone Lescano et al. (2016), sin embargo, una falencia en este enfoque es que no se consideran las fallas cognitivas de los estudiantes, lo que puede ser un factor determinante en la conformación de equipos de trabajo.

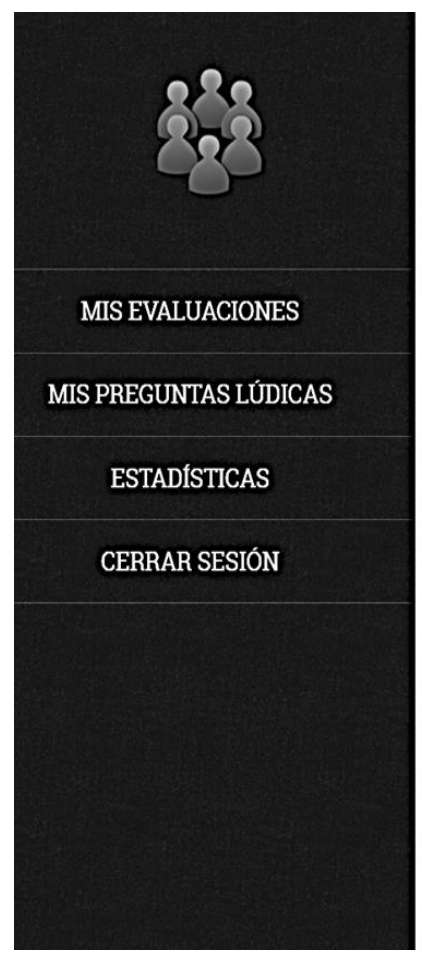

\section{Recomendación de recursos - Evaluación 2}

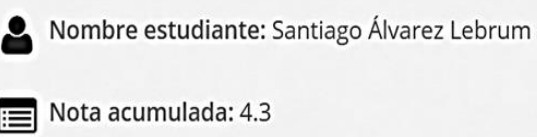

Fallas cognitivas diagnosticadas

Visualiza los recursos educativos recomendados para cada falla

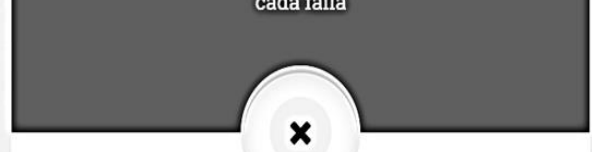

Distingue operaciones sobre conjuntos difusos

Entiende el método de inferencia difusa de Mamdani
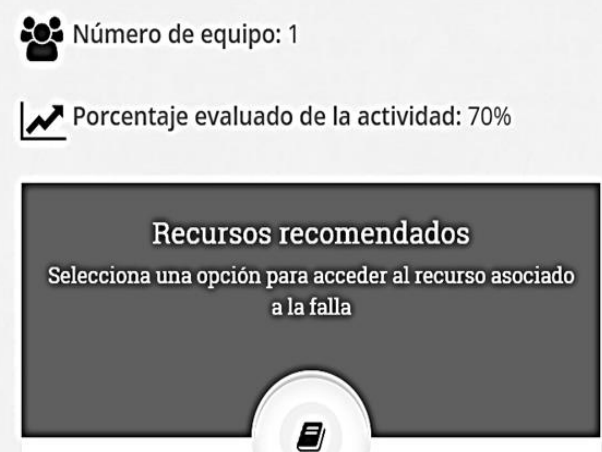

Video operaciones sobre conjuntos difusos

Diapositivas operaciones con conjuntos difusos

\section{Regresar}

Fig. 4: Interfaz de recomendación de recursos educativos del prototipo.

\section{CONCLUSIONES}

A partir de los resultados obtenidos con la implementación del modelo propuesto para la detección de fallas cognitivas durante el desarrollo de actividades colaborativas, y luego de la validación del prototipo se logra concluir que: 1) se demuestra la eficacia de la integración del esquema para el desarrollo de actividades colaborativas con cada una de las tecnologías propuestas en esta investigación; 2) se observó que los estudiantes se vieron más comprometidos y motivados al ser evaluados y retroalimentados de forma individual constantemente; 3) se evidenció tanto en las encuestas de los estudiantes como durante el desarrollo de la actividad colaborativa, que la actividad lúdica genera una interdependencia positiva entre los integrantes del equipo, lo cual los mantiene en un estado de alerta y de conciencia para estar atentos a cada uno de los detalles asociados a los conocimientos relacionados con los tópicos de la actividad.

\section{AGRADECIMIENTOS}

La investigación presentada en este artículo fue financiada parcialmente por la beca de Jóvenes investigadores de COLCIENCIAS, otorgada a Santiago Álvarez Lebrum a través de la Convocatoria 761 "Convocatoria Nacional de Jóvenes Investigadores e Innovadores 2016".

\section{REFERENCIAS}

Acevedo, C.P., J. L. Arciniegas, X. García y J. Perrinet, Proceso de Adaptación de una Aplicación de e-aprendizaje a taprendizaje, Información Tecnológica, 21 (6), 27-36 (2010)

Caicedo, C.E. y M.B. Weiss, The viability of spectrum trading markets, IEEE Communications Magazine, 49(3), 46-52 (2011)

Carrera, Á., C.A. Iglesias, J. García-Algarra y D. Kolařík, A real-life application of multi-agent systems for fault diagnosis in the provision of an Internet business service, Journal of Network and Computer Applications, 37, 146-154 (2014)

de Graaf, K.A., P. Liang, A. Tang, W.R. van Hage y H. van Vliet, An exploratory study on ontology engineering for software architecture documentation, Computers in Industry, 65(7), 1053-1064 (2014) 
Esnawy, S., EFL/EAP Reading and Research Essay Writing Using Jigsaw, Procedia-Social and Behavioral Sciences, 232, 98-101 (2016)

Fernández-López, M., A. Gómez-Pérez y N. Juristo, Methontology: from ontological art towards ontological engineering, Ontological Engineering, Vol. 06 of AAAI Spring Symposium, 33-40 (1997)

Herrera, R.F., F.C. Muñoz y L.A. Salazar, Diagnóstico del Trabajo en Equipo en Estudiantes de Ingeniería en Chile, Formación Universitaria, 10(5), 49-58 (2017)

Hübscher, R., Assigning Students to Groups Using General and Context-Specific Criteria, IEEE Transactions on Learning Technologies, 3(3), 178-189 (2010)

Lescano, G., R. Costaguta y A. Amandi, Genetic algorithm for automatic group formation considering student's learning styles, 8th Euro American Conference on Telematics and Information Systems (EATIS), 1-8 (2016)

Lhafiane, F., A. Elbyed y M. Bouchoum, Multi Agent System Architecture Oriented Prometheus Methodology Design for Reverse Logistics, World Academy of Science, Engineering and Technology, International Journal of Computer, Electrical, Automation, Control and Information Engineering, 9(8), 1914-1920 (2015)

Oshima, T., T. Mizuochi, K. Yatushiro y K. Mizukoshi, Development of CSCL System with Usability for Lower Elementary School Grade Students*, Proceedings of the 2016 IEEE/SICE International Symposium on System Integration, Sapporo Convention Center, Sapporo, Japan, 646-651 (2016)

Ovalle, D.A., O.M. Salazar y N.D. Duque, Modelo de Recomendación Personalizada en Cursos Virtuales basado en Computación Ubicua y Agentes Inteligentes, doi: 10.4067/S0718-07642014000600016, Información Tecnológica, 25 (6), 131-142 (2014)

Panisson, A.R., R.H. Bordini y A.C. da Rocha Costa, Towards Multi-Level Semantics for Multi-Agent Systems, Electronic Notes in Theoretical Computer Science, 324, 123-134 (2016)

Rodríguez, P.A., N.D. Duque y D.A. Ovalle, Método Híbrido de Recomendación Adaptativa de Objetos de Aprendizaje basado en Perfiles de Usuario, Formación Universitaria, 9(4), 83-94 (2016)

Rodríguez, P.A., V. Tabares, N.D. Duque, D.A. Ovalle y R.M. Vicari, BROA: An agent-based model to recommend relevant Learning Objects from Repository Federations adapted to learner profile, International Journal of Interactive Multimedia and Artificial Intelligence (2013)

Tudorache, T., C. Nyulas, N.F. Noy y M.A. Musen, WebProtégé: A collaborative ontology editor and knowledge acquisition tool for the web, Semantic web Journal, 4(1), 89-99 (2013)

Terzidou, T., T. Tsiatsos, C. Miliou y A. Sourvinou, Agent Supported Serious Game Environment, IEEE Transactions on Learning Technologies, 9(3), 217-230 (2016)

Valencia, C.A., P.A. Gaona-García, C.E. Montenegro-Marín y A.C. Gómez-Acosta, Ontological model based on web of trust to analyze the use of resources in learning environment, Iteckne, 14(1), 34-45 (2017) 
